Projektbereich B

Discussion Paper No. B-265

\title{
A Projection Result for Semimartingales
}

\author{
by \\ Martin Schweizer *)
}

January 1994

*) Financial support by Deutsche Forschungsgemeinschaft, Sonderforschungsbereich 303 at the University of Bonn, is gratefully acknowledged.

(Stochastics and Stochastics Reports 50 (1994), 175-183)

This version: 04.05.1995 


\title{
A Projection Result for Semimartingales
}

\author{
Martin Schweizer \\ Universität Göttingen \\ Institut für Mathematische Stochastik \\ Lotzestraße 13 \\ D-37083 Göttingen \\ Germany
}

Abstract: Let $X$ be a semimartingale and $\Theta$ the space of all predictable $X$-integrable processes $\vartheta$ such that $G(\vartheta):=\int \vartheta d X$ is in the space $\mathcal{S}^{2}$ of semimartingales. Assume that $X$ is special and has the form $X=X_{0}+M+\int \alpha d\langle M\rangle$. We show that for every fixed $T>0$, the space $G_{T}(\Theta)$ of stochastic integrals is closed in $\mathcal{L}^{2}$ if the process $\int \alpha^{2} d\langle M\rangle$ is bounded on $[0, T]$ and has jumps strictly bounded above by 1 . This allows us to solve a quadratic optimization problem arising in financial mathematics.

Key words: semimartingales, stochastic integrals, projection theorem, mean-variance tradeoff, financial mathematics

1991 Mathematics Subject Classification: 60G48, 60H05 


\section{Introduction}

If $M$ is a square-integrable martingale, then by its very construction, the stochastic integral with respect to $M$ is an isometry. For every fixed $T>0$, the space of stochastic integrals

$$
\left\{\int_{0}^{T} \vartheta_{s} d M_{s} \mid \int \vartheta d M \text { is a square-integrable martingale }\right\}
$$

is therefore a closed subspace of $\mathcal{L}^{2}$. In this paper, we extend this result to a certain class of $\mathbb{R}^{d}$-valued semimartingales. For ease of exposition, we formulate the results in the introduction only for $d=1$. We assume that $X$ is in $\mathcal{S}_{\text {loc }}^{2}$ and has a canonical decomposition of the form

$$
X=X_{0}+M+\int \alpha d\langle M\rangle \text {. }
$$

The process

$$
\widehat{K}_{t}:=\int_{0}^{t} \alpha_{s}^{2} d\langle M\rangle_{s} \quad, \quad 0 \leq t \leq T
$$

is called the mean-variance tradeoff process for $X$. Our main result then states that if $\widehat{K}_{T}$ is $P$-a.s. bounded and if

$$
\sup \left\{\widehat{K}_{\tau}-\widehat{K}_{\tau-} \mid \tau \text { is stopping time } \leq T P \text {-a.s. }\right\} \leq b<1 \quad P \text {-a.s. }
$$

for some constant $b$, then the space

$$
\left\{\int_{0}^{T} \vartheta_{s} d X_{s} \mid \int \vartheta d X \text { is a semimartingale in } \mathcal{S}^{2}\right\}
$$

is also closed in $\mathcal{L}^{2}$. This is rather remarkable since in contrast to the martingale case, stochastic integration with respect to a semimartingale is in general not an isometry. We point out that recent independent work by P. Monat and C. Stricker has shown that condition (0.1) is actually unnecessary; see Monat/Stricker [8,9] for details. On the other hand, a counterexample in section 3 illustrates that boundedness of $\widehat{K}$ is in general indispensable. As an immediate application, we obtain an existence result for a quadratic optimization problem arising in financial mathematics.

\section{Preliminaries}

Let $(\Omega, \mathcal{F}, P)$ be a probability space with a filtration $\mathbb{F}=\left(\mathcal{F}_{t}\right)_{0 \leq t \leq T}$ satisfying the usual conditions of right-continuity and completeness, where $T>0$ is a fixed and finite time horizon. For unexplained notation, terminology and results from martingale theory, we refer to Dellacherie/Meyer [3] and Jacod [6]. Without special mention, all processes will be defined for $t \in[0, T]$. Let $X$ be an $\mathbb{R}^{d}$-valued semimartingale in $\mathcal{S}_{\text {loc }}^{2}$; for the canonical decomposition

$$
X=X_{0}+M+A
$$


this means that $M \in \mathcal{M}_{0 \text {,loc }}^{2}$ and that the variation of the predictable finite variation part $A^{i}$ of $X^{i}$ is locally square-integrable for each $i$. We can and shall choose versions of $M$ and $A$ such that $M^{i}$ and $A^{i}$ are RCLL for each $i$. We shall assume that for each $i$,

$$
A^{i} \ll\left\langle M^{i}\right\rangle \quad \text { with predictable density } \alpha^{i} \text {. }
$$

Throughout the sequel, we fix a predictable locally integrable increasing RCLL process $B$ null at 0 such that $\left\langle M^{i}\right\rangle \ll B$ for each $i$. Since this implies $\left\langle M^{i}, M^{j}\right\rangle \ll B$ for all $i$, $j$, we can define the predictable matrix-valued process $\sigma$ by

$$
\sigma_{t}^{i j}:=\frac{d\left\langle M^{i}, M^{j}\right\rangle_{t}}{d B_{t}} \quad \text { for } i, j=1, \ldots, d
$$

We also define the predictable $\mathbb{R}^{d}$-valued process $\gamma$ by

$$
\gamma_{t}^{i}:=\alpha_{t}^{i} \sigma_{t}^{i i} \quad \text { for } i=1, \ldots, d
$$

so that for each $i$,

$$
A_{t}^{i}=\int_{0}^{t} \gamma_{s}^{i} d B_{s}
$$

Definition. The space $L^{2}(M)$ consists of all predictable $\mathbb{R}^{d}$-valued processes $\vartheta$ such that

$$
E\left[\int_{0}^{T} \vartheta_{s}^{*} \sigma_{s} \vartheta_{s} d B_{s}\right]<\infty
$$

where ${ }^{*}$ denotes transposition. The space $L^{2}(A)$ consists of all predictable $\mathbb{R}^{d}$-valued processes $\vartheta$ such that

$$
E\left[\left(\int_{0}^{T}\left|\vartheta_{s}^{*} \gamma_{s}\right| d B_{s}\right)^{2}\right]<\infty
$$

Finally, we set $\Theta:=L^{2}(M) \cap L^{2}(A)$.

Definition. We say that $X$ satisfies the structure condition $(S C)$ if there exists a predictable $\mathbb{R}^{d}$-valued process $\widehat{\lambda}$ such that

$$
\sigma_{t} \widehat{\lambda}_{t}=\gamma_{t} \quad P \text {-a.s. for all } t \in[0, T]
$$

and

$$
\widehat{K}_{t}:=\int_{0}^{t} \widehat{\lambda}_{s}^{*} \gamma_{s} d B_{s}<\infty \quad P \text {-a.s. for all } t \in[0, T] .
$$

We then choose an RCLL version of $\widehat{K}$ and call it the mean-variance tradeoff process of $X$. Note that these definitions imply that $\widehat{\lambda} \in L_{\text {loc }}^{2}(M)$ and

$$
\widehat{K}=\left\langle\int \widehat{\lambda} d M\right\rangle \text {. }
$$


Condition (SC) is naturally satisfied in most situations arising in financial mathematics; see Schweizer [14]. For $d=1$, we can choose $B:=\langle M\rangle$ and $\widehat{\lambda}:=\alpha=\gamma$; condition (SC) then follows from (1.1) and the assumption that $\alpha \in L_{\text {loc }}^{2}(M)$, and $\widehat{K}=\int \alpha^{2} d\langle M\rangle$.

For any $\vartheta \in \Theta$, the stochastic integral process $G(\vartheta):=\int \vartheta d X$ is well-defined and a semimartingale in $\mathcal{S}^{2}$ with canonical decomposition

$$
G(\vartheta)=\int \vartheta d M+\int \vartheta^{*} d A
$$

For our purposes, it is more convenient to use an alternative description of the space $\Theta$. If we denote by $L(X)$ the set of all $\mathbb{R}^{d}$-valued $X$-integrable predictable processes, then we have (as in Schweizer [13])

Lemma 1. If $X$ satisfies (1.1), then

$$
\Theta=\left\{\vartheta \in L(X) \mid \int \vartheta d X \in \mathcal{S}^{2}\right\}=: \Theta^{\prime}
$$

If in addition $X$ satisfies (SC) and $\widehat{K}_{T}$ is bounded, then $\Theta=L^{2}(M)$.

Proof. Since the variation of $\int \vartheta^{*} d A$ is given by $\int\left|\vartheta^{*} \gamma\right| d B$, it is clear that $\Theta^{\prime}$ contains $L^{2}(M) \cap L^{2}(A)$. Conversely, $X$ is special and $\int \vartheta d X$ is special for any $\vartheta \in \Theta^{\prime}$; hence $\int \vartheta d M$ and $\int \vartheta^{*} d A$ both exist in the usual sense by Théorème 2 of Chou/Meyer/Stricker [2], and $\int \vartheta d X \in \mathcal{S}^{2}$ thus implies that $\vartheta \in L^{2}(M) \cap L^{2}(A)$. Finally,

$$
\int_{0}^{T}\left|\vartheta_{s}^{*} \gamma_{s}\right| d B_{s} \leq \int_{0}^{T}\left(\vartheta_{s}^{*} \sigma_{s} \vartheta_{s}\right)^{\frac{1}{2}}\left(\widehat{\lambda}_{s}^{*} \sigma_{s} \widehat{\lambda}_{s}\right)^{\frac{1}{2}} d B_{s} \leq\left(\widehat{K}_{T}\right)^{\frac{1}{2}}\left(\int_{0}^{T} \vartheta_{s}^{*} \sigma_{s} \vartheta_{s} d B_{s}\right)^{\frac{1}{2}}
$$

shows that $L^{2}(M) \subseteq L^{2}(A)$ if $\widehat{K}_{T}$ is bounded.

q.e.d.

\section{The main result}

Let us now study in more detail the space $G_{T}(\Theta)$ of stochastic integrals.

Lemma 2. Suppose that $\tau, \tau^{\prime}$ are stopping times with $\tau \leq \tau^{\prime} \leq T P$-a.s. and

$$
\widehat{K}_{\tau^{\prime}}-\widehat{K}_{\tau} \leq c<1 \quad P \text {-a.s. for some constant } c \text {. }
$$

Then there exists a constant $C \in(0, \infty)$, depending only on $c$, such that for every $\vartheta \in \Theta$,

$$
E\left[\left(\int_{\tau}^{\tau^{\prime}} \vartheta_{s} d M_{s}\right)^{2}\right] \leq C E\left[\left(\int_{0}^{\tau^{\prime}} \vartheta_{s} d X_{s}\right)^{2}\right] .
$$


Proof. Choose $\varepsilon>0$ such that $(1+\varepsilon) c<1$. Write

$$
\begin{aligned}
\int_{\tau}^{\tau^{\prime}} \vartheta_{s} d M_{s} & =\int_{\tau}^{\tau^{\prime}} \vartheta_{s} d X_{s}-\int_{\tau}^{\tau^{\prime}} \vartheta_{s}^{*} d A_{s} \\
& =\int_{0}^{\tau^{\prime}} \vartheta_{s} d X_{s}-E\left[\int_{0}^{\tau^{\prime}} \vartheta_{s} d X_{s} \mid \mathcal{F}_{\tau}\right]-\left(\int_{\tau}^{\tau^{\prime}} \vartheta_{s}^{*} d A_{s}-E\left[\int_{\tau}^{\tau^{\prime}} \vartheta_{s}^{*} d A_{s} \mid \mathcal{F}_{\tau}\right]\right)
\end{aligned}
$$

and use the inequality

$$
(u-v)^{2} \leq\left(1+\frac{1}{\varepsilon}\right) u^{2}+(1+\varepsilon) v^{2}
$$

to obtain

$$
\begin{aligned}
(2.3) E\left[\left(\int_{\tau}^{\tau^{\prime}} \vartheta_{s} d M_{s}\right)^{2}\right] & \leq\left(1+\frac{1}{\varepsilon}\right) E\left[\operatorname{Var}\left[\int_{0}^{\tau^{\prime}} \vartheta_{s} d X_{s} \mid \mathcal{F}_{\tau}\right]\right] \\
& +(1+\varepsilon) E\left[\operatorname{Var}\left[\int_{\tau}^{\tau^{\prime}} \vartheta_{s}^{*} d A_{s} \mid \mathcal{F}_{\tau}\right]\right] \\
\leq & \left(1+\frac{1}{\varepsilon}\right) E\left[\left(\int_{0}^{\tau^{\prime}} \vartheta_{s} d X_{s}\right)^{2}\right]+(1+\varepsilon) E\left[\left(\int_{\tau}^{\tau^{\prime}} \vartheta_{s}^{*} d A_{s}\right)^{2}\right] .
\end{aligned}
$$

By (1.2), (1.4) and the Cauchy-Schwarz inequality,

$$
\left(\int_{\tau}^{\tau^{\prime}} \vartheta_{s}^{*} d A_{s}\right)^{2} \leq \int_{\tau}^{\tau^{\prime}} \vartheta_{s}^{*} \sigma_{s} \vartheta_{s} d B_{s} \int_{\tau}^{\tau^{\prime}} \widehat{\lambda}_{s}^{*} \sigma_{s} \widehat{\lambda}_{s} d B_{s}=\left(\widehat{K}_{\tau^{\prime}}-\widehat{K}_{\tau}\right) \int_{\tau}^{\tau^{\prime}} \vartheta_{s}^{*} \sigma_{s} \vartheta_{s} d B_{s},
$$

and so we conclude from (2.1) that

$$
E\left[\left(\int_{\tau}^{\tau^{\prime}} \vartheta_{s}^{*} d A_{s}\right)^{2}\right] \leq c E\left[\int_{\tau}^{\tau^{\prime}} \vartheta_{s}^{*} \sigma_{s} \vartheta_{s} d B_{s}\right]=c E\left[\left(\int_{\tau}^{\tau^{\prime}} \vartheta_{s} d M_{s}\right)^{2}\right] .
$$

Inserting this in (2.3) and rearranging yields (2.2), with

$$
C=\frac{1+\frac{1}{\varepsilon}}{1-(1+\varepsilon) c} \text {. }
$$

Theorem 3. Assume that $\widehat{K}_{T}$ is P-a.s. bounded by a constant and that

$$
\sup \left\{\widehat{K}_{\tau}-\widehat{K}_{\tau-} \mid \tau \text { is stopping time } \leq T P \text {-a.s. }\right\} \leq b<1 \quad P \text {-a.s. }
$$

for some constant $b$. Then $G_{T}(\Theta)$ is closed in $\mathcal{L}^{2}$. 
Proof. Thanks to the boundedness of $\widehat{K}_{T}$ and (2.5), we can find $N \in \mathbb{N}$ and stopping times $0=\tau_{0} \leq \tau_{1} \leq \ldots \leq \tau_{N}=T P$-a.s. such that

$$
\widehat{K}_{\tau_{j}}-\widehat{K}_{\tau_{j-1}} \leq c<1 \quad P \text {-a.s. for } j=1, \ldots, N \text { and some constant } c .
$$

Now suppose that $\left(G_{T}\left(\vartheta^{m}\right)\right)_{m \in \mathbb{N}}$ converges in $\mathcal{L}^{2}$ to some limit $Y$. Applying Lemma 2 with $\tau^{\prime}:=T$ and $\tau:=\tau_{N-1}$ shows that

$$
\left(\int_{\sigma_{N-1}}^{T} \vartheta_{s}^{m} d M_{s}\right)_{m \in \mathbb{N}} \text { is a Cauchy sequence in } \mathcal{L}^{2}
$$

Hence $\left(\vartheta^{m} I_{\rrbracket \tau_{N-1}, T \rrbracket}\right)_{m \in \mathbb{N}}$ is a Cauchy sequence in $L^{2}(M)$ and thus converges to $\psi^{N} I_{\left.\left.\rrbracket] \tau_{N-1}, T\right]\right]}$ for some $\psi^{N} \in L^{2}(M)$. Since $\widehat{K}_{T}$ is bounded, (2.4) yields

$$
E\left[\left(\int_{\tau}^{\tau^{\prime}} \vartheta_{s} d X_{s}\right)^{2}\right] \leq 2\left(1+\left\|\widehat{K}_{T}\right\|_{\infty}\right) E\left[\left(\int_{\tau}^{\tau^{\prime}} \vartheta_{s} d M_{s}\right)^{2}\right]
$$

for every $\vartheta \in \Theta$ and all stopping times $\tau \leq \tau^{\prime} \leq T P$-a.s. This implies that

$$
\int_{\tau_{N-1}}^{T} \vartheta_{s}^{m} d X_{s} \text { converges to } \int_{\tau_{N-1}}^{T} \psi_{s}^{N} d X_{s} \text { in } \mathcal{L}^{2}
$$

and therefore $\left(G_{\tau_{N-1}}\left(\vartheta^{m}\right)\right)_{m \in \mathbb{N}}$ converges in $\mathcal{L}^{2}$ to $Y-\int_{\tau_{N-1}}^{T} \psi_{s}^{N} d X_{s}$. Iterating this argument shows that

$$
Y=\int_{0}^{T} \vartheta_{s}^{\infty} d X_{s}
$$

with

$$
\vartheta^{\infty}:=\sum_{j=1}^{N} \psi^{j} I_{]] \tau_{j-1}, \tau_{j}\right]\right]}
$$

and since $\vartheta^{\infty}$ is clearly in $L^{2}(M)=\Theta$, the assertion follows.

q.e.d.

Remarks. 1) A simple modification of the proof of Lemma 2 yields the inequalities

$$
E\left[\left(\int_{\tau}^{\tau^{\prime}} \vartheta_{s} d M_{s}\right)^{2}\right] \leq C E\left[\left(\int_{\tau}^{\tau^{\prime}} \vartheta_{s} d X_{s}\right)^{2}\right] \leq 2 C(1+c) E\left[\left(\int_{\tau}^{\tau^{\prime}} \vartheta_{s} d M_{s}\right)^{2}\right]
$$

This provides the intuition behind the closedness result in Theorem 3: under the assumptions made there,

$$
\left\|\int_{0}^{T} \vartheta_{s} d X_{s}\right\|_{\mathcal{L}^{2}} \text { and }\left\|\int_{0}^{T} \vartheta_{s} d M_{s}\right\|_{\mathcal{L}^{2}}=\|\vartheta\|_{L^{2}(M)}
$$


are essentially equivalent norms on $\Theta$, and so the semimartingale case considered here is not too far away from the martingale case. For a proof that the above two norms are actually equivalent, see Monat/Stricker [9].

2) It is interesting to note that the conditions of Theorem 3 are exactly the same as those guaranteeing the existence of a strong F-S decomposition for $\mathcal{F}_{T}$-measurable random variables $H \in \mathcal{L}^{2}$; see Schweizer [13]. We also point out that the above proof is analogous to the argument for the discrete-time case treated in Schweizer [12].

3) After this paper was submitted, we learnt from C. Stricker that he and P. Monat had independently also proved the closedness of $G_{T}(\Theta)$, even without assuming condition (2.5). Their argument rests on showing that the strong F-S decomposition of a square-integrable random variable exists and is unique and continuous; see Monat/Stricker [8]. In a subsequent paper, Monat/Stricker [9] then showed how to modify the direct argument of the present paper in order to eliminate assumption (2.5). In both cases, the essential step is to use the predictability of $\vartheta, A$ and $\widehat{K}$ in a suitable way.

\section{Applications and examples}

Apart from condition (2.5), Theorem 3 is the best possible result. The following example due to W. Schachermayer shows that $G_{T}(\Theta)$ need not be closed in $\mathcal{L}^{2}$ if $\widehat{K}_{T}$ is unbounded. For simplicity, we formulate the example in discrete time; choosing piecewise constant RCLL processes and a piecewise constant right-continuous filtration immediately yields a continuoustime version. For a similar example with a continuous process $X$, see Monat/Stricker [8].

Example. Let $S, U$ be independent with $U$ uniform on $[0,1]$ and the distribution of $S$ nondegenerate with finite second moment. Given $U$, the random variable $V$ takes the values \pm 1 with respective probabilities $U^{2}, 1-U^{2}$. Take $T=2$ and define the discrete-time process $\left(X_{k}\right)_{k=0,1,2}$ by setting $X_{0}=0, X_{1}=S$ and $X_{2}=(S+U) V^{+}$. The filtration $\left(\mathcal{F}_{k}\right)_{k=0,1,2}$ is given by $\mathcal{F}_{0}=\sigma(U), \mathcal{F}_{1}=\sigma(U, S)$ and $\mathcal{F}_{2}=\sigma(U, S, V)$. Then we get

$$
\begin{aligned}
\widehat{K}_{1} & =\frac{(E[S])^{2}}{\operatorname{Var}[S]}, \\
\widehat{K}_{2} & =\frac{\left(U^{3}+S U^{2}-S\right)^{2}}{U^{2}(U+S)^{2}-U^{5}(U+2 S)},
\end{aligned}
$$

and as $U$ approaches 0 , the last ratio tends to infinity so that $\widehat{K}_{2}$ is unbounded in $\omega$.

Now consider the sequence of predictable processes

$$
\vartheta^{n}=\frac{1}{U} I_{\left\{U \geq \frac{1}{n}\right\}}
$$

Then

$$
\begin{aligned}
& G_{1}\left(\vartheta^{n}\right)=\frac{S}{U} I_{\left\{U \geq \frac{1}{n}\right\}} \in \mathcal{L}^{2}, \\
& G_{2}\left(\vartheta^{n}\right)=\frac{1}{U}(S+U) V^{+} I_{\left\{U \geq \frac{1}{n}\right\}} \in \mathcal{L}^{2},
\end{aligned}
$$

and so $\vartheta^{n} \in \Theta$ for all $n$. Moreover, it is evident that $G_{2}\left(\vartheta^{n}\right)$ converges in $\mathcal{L}^{2}$ to

$$
H=\frac{1}{U}(S+U) V^{+} \in \mathcal{L}^{2} .
$$


But the only predictable process $\xi$ with $G_{2}(\xi)=H$ is $\xi=\frac{1}{U}$ (consider the sets $\{V= \pm 1\}$ ), and since

$$
G_{1}(\xi)=\frac{S}{U} \notin \mathcal{L}^{2}
$$

$\xi$ is not in $\Theta$, so $H$ is not in $G_{2}(\Theta)$ and $G_{2}(\Theta)$ is not closed in $\mathcal{L}^{2}$. This ends the example.

As an immediate consequence of Theorem 3, we get

Corollary 4. If $\widehat{K}_{T}$ is P-a.s. bounded and satisfies (2.5), there exists a unique solution $\xi^{(c)} \in \Theta$ to the problem

$$
\text { Minimize } E\left[\left(H-c-\int_{0}^{T} \vartheta_{s} d X_{s}\right)^{2}\right] \text { over all } \vartheta \in \Theta
$$

for every pair $(H, c) \in \mathcal{L}^{2} \times \mathbb{R}$.

This result answers a previously unresolved question from financial mathematics concerning the existence of a variance-minimizing hedging strategy. Except for the case of finite discrete time completely solved in Schweizer [12], earlier work on this problem was all based on very restrictive assumptions; see Duffie/Richardson [4], Schäl [10], Schweizer [11], Hipp [5], Schweizer [13]. For a slightly more general result, we refer to Monat/Stricker [8]. Like Theorem 3, Corollary 4 is almost sharp: the same example as above shows that (3.1) need not have a solution in general.

A second application concerns the problem of variance-minimization under restricted information. For any subfiltration $\mathbb{G} \subseteq \mathbb{F}$ satisfying the usual conditions, denote by $\Theta(\mathbb{G})$ the set of those $\vartheta \in \Theta$ which are $\mathbb{G}$-predictable. In answer to a question of D. Heath, we then have

Theorem 5. If $\widehat{K}_{T}$ is $P$-a.s. bounded and satisfies (2.5), then $G_{T}(\Theta(\mathbb{G}))$ is closed in $\mathcal{L}^{2}$ for any filtration $\mathbb{G} \subseteq \mathbb{F}$ satisfying the usual conditions.

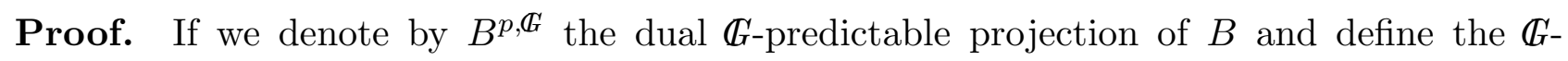
predictable processes

$$
\varrho^{i j}:=\frac{d\left(\int \sigma^{i j} d B\right)^{p, G_{r}}}{d B^{p, G_{r}}} \quad \text { for } i, j=1, \ldots, d
$$

then (1.3) can be rewritten as

$$
E\left[\int_{0}^{T} \vartheta_{s}^{*} \varrho_{s} \vartheta_{s} d B_{s}^{p, G}\right]<\infty
$$

This shows that $\Theta(G)$ is closed in $L^{2}(M)$, and so the assertion follows as in Theorem 3 .

$$
\text { q.e.d. }
$$


Corollary 6. If $\widehat{K}_{T}$ is P-a.s. bounded and satisfies (2.5), there exists a unique solution to

$$
\text { Minimize } E\left[\left(H-c-\int_{0}^{T} \vartheta_{s} d X_{s}\right)^{2}\right] \text { over all } \vartheta \in \Theta(\mathbb{G})
$$

for every pair $(H, c) \in \mathcal{L}^{2} \times \mathbb{R}$ and every filtration $\mathbb{G} \subseteq \mathbb{F}$ satisfying the usual conditions.

We conclude this paper with some examples where the assumptions of Theorem 3 are satisfied. Of course, (2.5) is trivially fulfilled whenever $\widehat{K}$ is continuous. This is always the case if $X$ is continuous, so that it then only remains to check boundedness of $\widehat{K}$. (Actually, this is always sufficient; see Monat/Stricker [8,9].) Another example is provided by the multidimensional jump-diffusion model considered in Shirakawa $[15,16]$, Xue [17] and Schweizer [13], among others. As a special case, this includes the multidimensional diffusion model introduced by Bensoussan [1] and studied for instance by Karatzas/Lehoczky/Shreve/Xue [7]. In the one-dimensional case, this model reduces to

$$
d X_{t}=\mu_{t} X_{t} d t+\sigma_{t} X_{t} d W_{t}
$$

and since

$$
\widehat{K}_{t}=\int_{0}^{t} \frac{\mu_{s}^{2}}{\sigma_{s}^{2}} d s,
$$

Corollary 4 gives an existence result for $\left(\mu_{t} / \sigma_{t}\right)$ bounded. This is a clear improvement over previous results which typically required this ratio to be deterministic; see Schweizer [11].

Acknowledgement. This work was completed during a visit to the Australian National University in Canberra. Financial support by Deutsche Forschungsgemeinschaft, Sonderforschungsbereich 303 at the University of Bonn, is gratefully acknowledged. I also thank Christophe Stricker for sending me the preprints Monat/Stricker [8,9].

\section{References}

[1] A. Bensoussan (1984), "On the Theory of Option Pricing", Acta Applicandae Mathematicae 2, 139-158

[2] C. S. Chou, P.-A. Meyer and C. Stricker (1980), "Sur les Intégrales Stochastiques de Processus Prévisibles Non Bornés", Séminaire de Probabilités XIV, Lecture Notes in Mathematics 784, Springer, 128-139

[3] C. Dellacherie and P.-A. Meyer (1982), "Probabilities and Potential B", North-Holland

[4] D. Duffie and H. R. Richardson (1991), "Mean-Variance Hedging in Continuous Time", Annals of Applied Probability 1, 1-15

[5] C. Hipp (1993), "Hedging General Claims", Proceedings of the 3rd AFIR Colloquium, Rome, Vol. 2, 603-613

[6] J. Jacod (1979), "Calcul Stochastique et Problèmes de Martingales", Lecture Notes in Mathematics 714, Springer 
[7] I. Karatzas, J. P. Lehoczky, S. E. Shreve and G.-L. Xu (1991), "Martingale and Duality Methods for Utility Maximization in an Incomplete Market", SIAM Journal on Control and Optimization 29, 702-730

[8] P. Monat and C. Stricker (1993), "Föllmer-Schweizer Decomposition and Closedness of $G_{T}(\Theta)$ ", preprint, Université de Franche-Comté, Besançon

[9] P. Monat and C. Stricker (1993), "Fermeture de $G_{T}(\Theta)$ et de $\left\{c+G_{T}(\Theta) \mid c \in \mathbb{R}\right\}$ ", preprint, Université de Franche-Comté, Besançon

[10] M. Schäl (1992), "On Quadratic Cost Criteria for Option Hedging", preprint, University of Bonn, to appear in Mathematics of Operations Research

[11] M. Schweizer (1992), "Mean-Variance Hedging for General Claims", Annals of Applied Probability 2, 171-179

[12] M. Schweizer (1993a), "Variance-Optimal Hedging in Discrete Time", preprint, University of Göttingen, to appear in Mathematics of Operations Research

[13] M. Schweizer (1993b), "Approximating Random Variables by Stochastic Integrals", preprint, University of Göttingen, to appear in Annals of Probability

[14] M. Schweizer (1993c), "On the Minimal Martingale Measure and the Föllmer-Schweizer Decomposition", preprint, University of Göttingen, to appear in Stochastic Analysis and Applications

[15] H. Shirakawa (1990a), "Security Market Model with Poisson and Diffusion Type Return Process", preprint IHSS 90-18, Tokyo Institute of Technology

[16] H. Shirakawa (1990b), "Optimal Dividend and Portfolio Decisions with Poisson and Diffusion Type Return Processes", preprint IHSS 90-20, Tokyo Institute of Technology

[17] X.-X. Xue (1992), "Martingale Representation for a Class of Processes with Independent Increments and its Applications", in: I. Karatzas and D. Ocone (eds.), "Applied Stochastic Analysis", Proceedings of a US-French Workshop, Rutgers University, New Brunswick, N.J., Lecture Notes in Control and Information Sciences 177, Springer, 279311 